\title{
Sistema de Información de los Indicadores de Seguridad Alimentaria y Nutricional del Departamento de Caldas
}

\author{
The Information System for the Food and Nutrition Security Indicators of the \\ Department of Caldas
}

\author{
D. P. Soto -Alzate iD
}

\begin{abstract}
This article presents the development of the Information System Indicators of Food Security and Nutritional Status of the Department of Caldas, carried out with the Food Security Network (SAN) experts and eighth semester software engineering students from the engineering and computing program at the University of Caldas 2013, as academic practice in which the judgment of experts applies, Personal Software Process (PSP) and Team Software Process introduction (TSPi) The study of the indicators of the United Nations Food and Agriculture Organization (FAO) and the indicators considered by the National Council of Social Economic Policy (CONPES) for the National Policy on Food Security and Nutrition (PSAN). A first list of 389 indicators is presented to the experts of which 27 of them which are required and monitored by the Nation's Attorney General Office are selected by consensus and require significant economic resources, which should be part of the Department's budget in order to implement other indicators. It was concluded that the judgment of experts helped to determine the indicators implemented and that the development processes TSPi and PSP requires discipline on Time registering, because students were not used to measuring each activity's time, however, the total of hours for the project were estimated and it was also determined that the organization and communication between the teams should be very good for the success of the same.
\end{abstract}

Index Terms - Indicators of food and nutritional security, judgment of experts, PSP TSPi

Resumen- El artículo presenta el desarrollo del Sistema de Información de los Indicadores de Seguridad Alimentaria y Nutricional del Departamento de Caldas, realizado con los expertos de la Red de Seguridad Alimentaria (SAN) y estudiantes de la asignatura ingeniería de software de octavo semestre de 2013 del programa de ingeniería y computación de la Universidad de Caldas, práctica académica en la que se aplica el juicio de expertos, Personal Software Process (PSP) y Team Software Process introduction (TSPi). Se realiza el estudio de los indicadores de la Organización de las Naciones Unidas para la Alimentación y la Agricultura (FAO) y los indicadores considerados por el Consejo Nacional de Política Económica Social (CONPES) para la Política Nacional de Seguridad Alimentaria y Nutricional (PSAN). Se presenta a los expertos un primer listado de 389 indicadores de los cuales son seleccionados en consenso 27 de ellos que son

El artículo de investigación es derivado de la tesis de grado del postgrado Maestría en Gestión y Desarrollo de Proyectos de Software de la Universidad Autónoma de Manizales. exigidos y vigilados por la Procuraduría General de la Nación y que para implementar otros indicadores se requiere de recursos económicos importantes los cuales deben ser parte del presupuesto del Departamento. Se concluye que el juicio de expertos ayudó a determinar los indicadores implementados y que los procesos de desarrollo TSPi y PSP requieren disciplina para el registro de tiempos, ya que los estudiantes no estaban acostumbrados a medir el tiempo de cada actividad, sin embargo, se pudo estimar el total de horas del proyecto y se determina también que la organización y comunicación entre los equipos debe ser muy buena para el éxito del mismo.

Palabras claves- Indicadores de seguridad alimentaria y nutricional, juicio de expertos, PSP, TSPi.

\section{INTRODUCTION}

$\mathbf{E}_{\text {to }}^{\mathrm{X}}$ XISTE seguridad alimentaria cuando las personas tienen en todo momento acceso físico y económico a suficientes alimentos inocuos y nutritivos para satisfacer sus necesidades alimenticias y sus preferencias en cuanto a los alimentos a fin de llevar una vida activa y sana. [1].

La seguridad alimentaria y nutricional (SAN) hace parte de una situación dinámica que abarca factores socioeconómicos, alimentarios, nutricionales y ambientales; de tal manera para que la población garantice su SAN, se debe realizar un análisis profundo de múltiples variables que logren el reconocimiento de la situación alimentaria y nutricional, a fin de establecer acciones documentadas y con soporte metodológico científico y sistematizado, para asegurar el logro de las metas y objetivos propuestos en beneficio de la población.

En el Departamento de Caldas en Colombia existe el Observatorio de Seguridad Alimentaria y Nutricional (OBSAN), el cual cuenta con la participación activa de la Caja de Compensación Familiar de Caldas (Confa), el Instituto Colombiano de Bienestar Familiar, la Dirección Territorial de Salud de Caldas, la Gobernación de Caldas y la Universidad de Caldas. El OBSAN es un espacio de reflexión, análisis, medición y monitoreo de las acciones y problemáticas SAN, soportado en la política pública para la construcción y difusión de conocimiento útil en la gestión de las soluciones respectivas,

D. P. Soto -Álzate, Docente Fundación Universitaria Comfamiliar. (e-mail. derly.soto@uc.edu.co 
es allí donde nace la necesidad de crear un sistema de información que permita unificar los criterios de evaluación, seguimiento y control de los indicadores de seguridad alimentaria y nutricional.

Este sistema de información se realizó con participación de los estudiantes de octavo semestre de la asignatura Ingeniería de Software III de la Universidad de Caldas, donde se aplicaron los procesos de desarrollo Introductory Team Software Process-TSPi (Introducción al Proceso de Software de Equipos) [2], y Personal Software Process-PSP (Proceso Personal de Software) [3]. El proyecto se realizó como práctica académica en el aula de clase, con el fin de enriquecer los procesos de desarrollo de software a partir de un proyecto real.

El presente documento describe el proceso de investigación aplicada y de ingeniería de software que permitieron la construcción del sistema de información de los indicadores de SAN.

\section{METODOLOGÍA}

\section{A. Método Delphi aplicado al juicio de expertos para la selección de indicadores de seguridad alimentaria.}

Una Delphi consiste en la selección de un grupo de expertos a los que se les pregunta su opinión sobre cuestiones referidas a acontecimientos del futuro. Las estimaciones de los expertos se realizan en sucesivas rondas anónimas, el objeto es tratar de conseguir consenso, pero con la máxima autonomía por parte de los participantes [4].

El juicio de expertos fue aplicado para llegar a un consenso de los indicadores que fueron implementados en el sistema de información; para tal fin se tomaron en cuenta los criterios de los actores que conforman la Red SAN del departamento de Caldas.

\section{1) Elaboración y lanzamiento de los cuestionarios}

Se creó una matriz donde se consolido un total de 389 indicadores de SAN, a nivel internacional se tomó en cuenta los presentados por las Naciones Unidas y la Unión Europea [5], a nivel nacional fueron tenidos en cuenta los del Ministerio de Salud y el Departamento Nacional de Planeación [6]. El consolidado final de indicadores fue enviado y socializado con los expertos.

\section{2) Informe}

Para este fin se realizó una matriz con los 197 indicadores que fueron seleccionados por los expertos, quienes concluyen que aunque todos son pertinentes para la seguridad alimentaria del departamento de Caldas, no se cuenta en el momento con la infraestructura técnica y recursos económicos para poder hacer seguimiento, control y evaluación de algunos de ellos.

Los expertos propusieron que se tuvieran en cuenta 27 indicadores que hacen referencia directa a la SAN de los 65 exigidos por la Procuraduría General de la Nación y de los cuales las entidades territoriales deben rendir informes, por hacer parte de la política pública del país ya que son de obligatorio cumplimiento.

B. El lenguaje de modelamiento unificado (uml - unified modeling language)
Lenguaje gráfico para visualizar, especificar y documentar cada una de las partes que comprende el desarrollo de software [7].

Los elementos gráficos que se aplicaron para modelar el sistema de indicadores SAN son: diagrama de casos de uso, diagrama de clases, diagrama de secuencia y diagrama de paquetes.

\section{Introductory Team Software Process (TSPi)}

La Universidad de Carnegie Mellon, en el Instituto de Ingeniería de Software (SEI) creó el proceso de desarrollo Team Software Process (TSP), que ayuda a las organizaciones a desarrollar software con calidad mediante la gestión de equipos de ingenieros de software. Derivado de éste se desarrolla la versión académica TSPi, que introduce a los ingenieros de software a desarrollar en equipos un proceso definido y establecido para producir productos de alta calidad. [8].

TSPi Considera siete características de diseño de un proceso: proporciona un marco simple que se basa en el fundamento de PSP; desarrolla productos en varios ciclos; establece medidas estándar de calidad y rendimiento; proporciona medidas precisas para los equipos y los estudiantes o ingenieros de software; evalúa roles y equipos; requiere una disciplina de proceso y promueve un marco de trabajo y guías.

\section{Personal Software Process (PSP)}

PSP brinda a los ingenieros la posibilidad de mejorar su desarrollo de software estableciendo un marco de disciplina personal. El proceso PSP consiste en un conjunto de métodos, formas que muestran como planificar, medir y administrar su propio trabajo.

En PSP todas las tareas y actividades están puntualmente definidas en un conjunto de documentos conocidos como scripts, los cuales son adoptados por cada estudiante para el registro de datos.

\section{PROCESO DE DESARROLLO}

\section{A. Lanzamiento}

Se realizó el lanzamiento del producto con 17 estudiantes de la asignatura ingeniería de software, se explicaron las necesidades del cliente, se definieron los objetivos del producto y del negocio, se establecieron los riesgos potenciales, se presentaron las actas de lanzamiento, se conformaron tres equipos de trabajo, se asignaron los roles de acuerdo al estudio de habilidades, se establecieron las metas y objetivos de cada rol y de cada equipo.

\section{1) Objetivos del Producto}

Desarrollar el sistema de indicadores SAN del departamento de Caldas, el cual consiste en crear la hoja de vida de los indicadores, registrar, evaluar los valores de las variables, computar valores y reportar los indicadores.

\section{2) Objetivos del Negocio}

La red SAN a partir de la implementación del sistema tiene la posibilidad de obtener la información y hacer seguimiento de los indicadores de seguridad alimentaria y nutricional del departamento de Caldas de manera segura y confiable, a través 
de un criterio unificado para todas las entidades adscritas, optimizando de esta manera el proceso de toma de decisiones.

\section{3) Objetivos del proyecto}

Cumplir con los requerimientos definidos en el documento de análisis de requerimientos.

\section{4) Meta del proyecto}

Implementar el > 90\% de los requerimientos establecidos.

\section{5) Objetivos de planeación}

Producir un plan completo, preciso y exacto del equipo y cada uno de sus integrantes.

\section{6) Meta de planeación}

Asignar tareas y un horario definido para su respectivo cumplimiento $>60 \%$.

\section{B. Estrategia}

El sistema de indicadores SAN se realizó con 17 estudiantes de la Universidad de Caldas de la asignatura Ingeniería de Software III del año 2013, los estudiantes se dividieron en tres grupos. El sistema se dividió en tres subsistemas, a cada grupo se le asignó uno de ellos y a cada subsistema se le realizó un ciclo de desarrollo, los tres subsistemas se realizaron de forma paralela y la integración del sistema se realizó con los líderes de desarrollo de cada grupo.

\section{Planeación}

\section{1) Planeación Equipo}

Se realizó la planeación de cada subsistema de acuerdo a las actividades, roles y requerimientos del cliente, la planeación de los componentes se realizó por número de horas.

\section{2) Planeación PSP}

Se asignaron las actividades a cada uno de los integrantes, se registró el plan en horas y el tiempo real en horas.

\section{3) Requerimientos PSP}

Cada uno de los estudiantes verificó los casos de uso y la especificación de casos de uso que le fue asignada.

\section{4) Revisar e Inspeccionar Requerimientos}

Se realizó la inspección a través de listas de chequeo, estos formatos se aplicaron para controlar el cumplimiento de una lista de requisitos, además de dejar trazabilidad de la actividad realizada.

\section{5) Registro Tiempos y defectos}

Cada integrante registró el tiempo dedicado a la planeación, defectos inyectados y eliminados.

\section{Requerimientos}

Para la obtención de requisitos se realizaron entrevistas y reuniones con los expertos del OBSAN, se consultaron indicadores internacionales y nacionales, la política pública de SAN a nivel local y departamental.

Se consultaron los indicadores exigidos por la Procuraduría General de la Nación [9]. Hoja de vida de los 65 indicadores, la rendición pública de cuentas presentada por la Gobernación de Caldas tomo I [10], Tomo II [11] y tomo [12], donde se presenta el estado de la seguridad alimentaria y nutricional, entre los años 2005 y 2010.

Consulta la política de seguridad alimentaria y nutricional de Colombia [13] y la política de seguridad alimentaria y nutricional de Caldas [14].

Consulta de indicadores internacionales presentados por la FAO y los indicadores presentados por la Unión Europea (Sistema regional de indicadores de seguridad alimentaria y nutricional (SIRSAN)).

Por parte de los expertos del OBSAN se define el formato de hoja de vida de los indicadores presentado por Planeación Nacional.

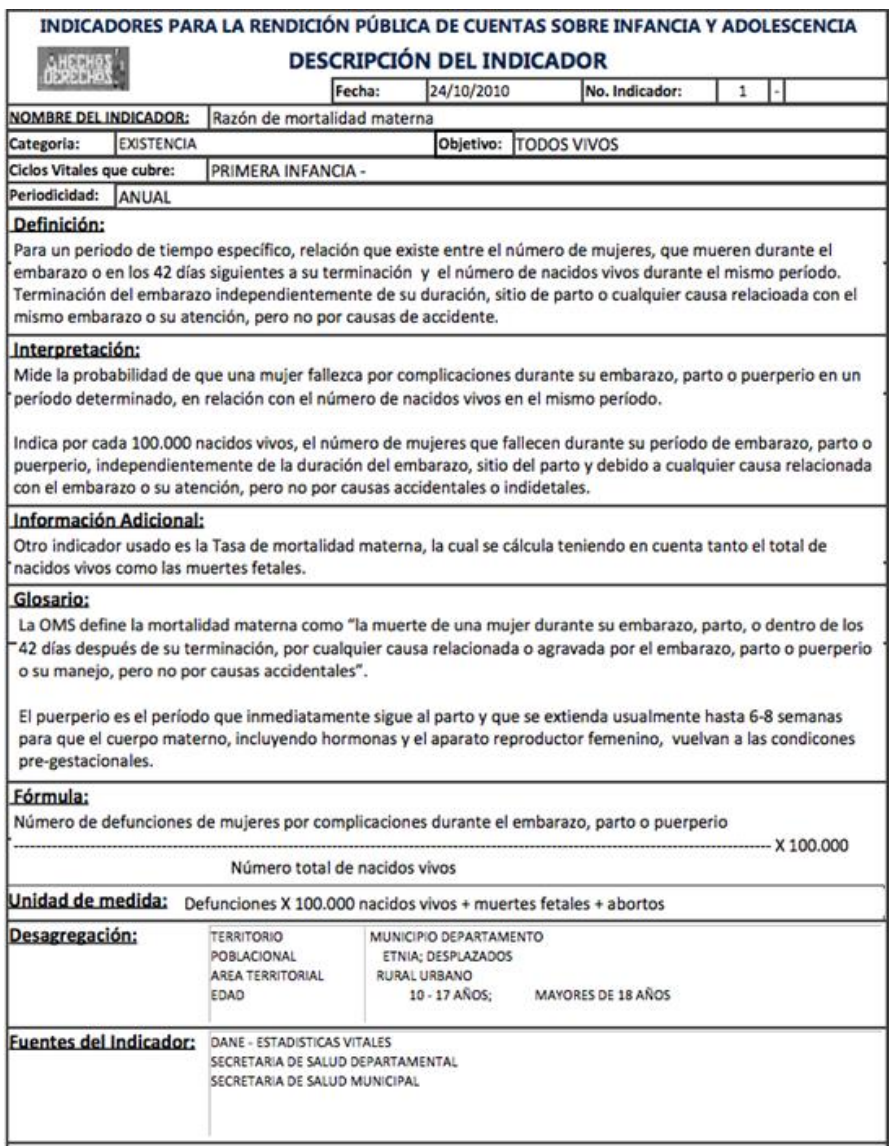

Fig 1. Formato Hoja de Vida del Indicador Fuente Procuraduría General de la Nación - 2011

\section{1) Requerimientos funcionales}

Gestionar la hoja de vida del indicador de acuerdo a las categorías, variables, ciclo vital y entidades responsables.

Registrar los valores de las variables, las enfermedades más frecuentes y responsables del indicador.

Consolidar indicadores por rangos de tiempo a nivel municipal y departamental de acuerdo a la fórmula definida para cada indicador

Evaluar el indicador a nivel municipal y departamental de acuerdo a los resultados de los datos consolidados. 


\section{2) Requerimientos no funcionales}

Java Server Pages (JSP), entorno de desarrollo NetBeans IDE 7.2.1, lenguaje de programación JAVA, motor de bases de datos Postgres 9.1, Process Dashboard para el registro de tiempos PSP, Herramientas de modelado UML - Staruml 5.0.2.1, Balsamic Mockups para los prototipos de pantalla, Herramientas Colaborativas Wiki, Google Drive, JaspersofIreports 5.1 para los reportes.

Características servidor de OBSAN, donde se instaló aplicación: Procesador IntelXeon 4 x $2.5 \mathrm{GHz}$, Memoria 8GB RAM, Disco Duro: 600GB en RAID 1, espacio asignado para el sistema de indicadores $=30 \mathrm{~GB}$.

Aunque PSP 0.1 no contempla las listas de chequeo se utilizaron formatos para verificar los entregables del análisis y el diseño [15].

\section{E. Diseño del sistema}

Se realizó la arquitectura a partir de la especificación detallada de los componentes del sistema de información y se implementa el patrón de diseño Modelo Vista Controlador (MVC).

Se presenta el uso de las convenciones de programación en Java, estándar de codificación para bases de datos, estándares de nombramiento para paquetes, clases, atributos y métodos, se realizaron los diagramas de clases, diagrama de paquetes, diseño de pantalla, se inspeccionaron las clases, diagramas de secuencia y la arquitectura del sistema a través de listas de chequeo, se diseña la base de datos y se hace el registro de tiempo de la fase del diseño, defectos inyectados y removidos.

\section{F. Implementación}

Se crea el producto en el lenguaje de programación Java, con el motor de base de datos postgres. Se produce el código de acuerdo con el estándar de codificación definido por el equipo, y se integran todos los módulos producidos durante la fase de implementación.

Se presenta el registro de líneas de código de cada subsistema de acuerdo a los casos de uso asignados y se registran los tiempos de la codificación.

\section{G. Pruebas del sistema}

\section{1) Pruebas de unidad}

A nivel de PSP cada estudiante verificó la funcionalidad y estructura de cada componente individualmente, probando los bloques más pequeños del módulo hasta llegar a su totalidad, antes de ser integrada al sistema.

\section{2) Pruebas funcionales}

Se realizaron pruebas funcionales para cada caso de uso con información real, suministrada por la Secretaría de Planeación de la Gobernación de Caldas, logrando con esto comparar los resultados de los indicadores del sistema con los presentados en los informes de rendición de cuentas, y de esta manera comprobar que el sistema presentara los resultados esperados.

\section{H. Postmortem TSPi y PSP}

Se presenta el registro de tiempos de los equipos, la propuesta de mejora, la evaluación de los roles, problemas encontrados en el grupo, plan de mejora de los equipos, objetivos y metas cumplidas, registro de los tiempos individuales y consolidación los tiempos del equipo. Registro de los defectos inyectados y removidos durante la fase de desarrollo.

\section{RESULTADOS}

Implementación del sistema de indicadores de seguridad alimentaria y nutricional del Departamento de Caldas, instalado en el servidor de OBSAN de la Universidad de Caldas.

El sistema presenta las variables y el estado de los indicadores, permite que los expertos evalúen indicadores, siendo este un gran aporte a la SAN en el Departamento para la toma de decisiones.

El estudio de indicadores de SAN a nivel internacional, nacional y departamental, logrando con ello crear un listado con los indicadores de la FAO, la ONU, Ministerio de Salud, Planeación Nacional y los 65 indicadores exigidos por la política pública.

Con los integrantes de la Red SAN se logró establecer los requerimientos, la hoja de vida de los indicadores, seleccionar los siguientes 27 indicadores los cuáles fueron implementados en el sistema de información.

TABLA I

INDICADORES DE SEGURIDAD ALIMENTARIA Y NUTRICIONAL SELECCIONADOS POR LOS EXPERTOS DE SAN

\begin{tabular}{|c|c|}
\hline Nro. & Indicador \\
\hline 1 & Razón de mortalidad materna \\
\hline 2 & Tasa de mortalidad en menores de 1 año \\
\hline 3 & Tasa de mortalidad de niños, niñas de 0 a 5 \\
\hline 4 & $\begin{array}{l}\text { Cinco primeras causas de mortalidad de niños, niñas entre los } 0 \text { y } \\
5 \text { años }\end{array}$ \\
\hline 5 & $\begin{array}{l}\text { Tasa de mortalidad de } 0 \text { a } 17 \text { años por causas externas } \\
\text { (homicidio, suicidio, accidentes, violencia intrafamiliar) }\end{array}$ \\
\hline 6 & $\begin{array}{l}\text { Porcentaje de niños, niñas y adolescentes valorados con } \\
\text { desnutrición crónica }\end{array}$ \\
\hline 7 & $\begin{array}{l}\text { Porcentaje de niños, niñas y adolescentes valorados con } \\
\text { desnutrición global }\end{array}$ \\
\hline 8 & $\begin{array}{l}\text { Porcentaje de niños, niñas entre } 0-6 \text { meses que asisten a controles } \\
\text { de crecimiento y desarrollo y que reciben lactancia materna } \\
\text { exclusiva }\end{array}$ \\
\hline 9 & $\begin{array}{l}\text { Porcentaje de mujeres gestantes con diagnóstico de anemia } \\
\text { nutricional }\end{array}$ \\
\hline 10 & Porcentaje de niños, niñas con bajo peso al nacer \\
\hline 11 & $\begin{array}{l}\text { Cobertura de inmunización contra el BCG en niños, niñas } \\
\text { menores de un año }\end{array}$ \\
\hline 12 & $\begin{array}{l}\text { Cobertura de inmunización contra el polio en niños y niñas } \\
\text { menores de } 1 \text { año }\end{array}$ \\
\hline 13 & $\begin{array}{l}\text { Cobertura de inmunización contra el DPT en niños y niñas } \\
\text { menores de } 1 \text { años }\end{array}$ \\
\hline 14 & $\begin{array}{l}\text { Cobertura de inmunización contra la Hepatitis B en niños y niñas } \\
\text { menores de } 1 \text { años }\end{array}$ \\
\hline 15 & $\begin{array}{l}\text { Cobertura de inmunización contra el Rotavirus en niños y niñas } \\
\text { menores de } 1 \text { año }\end{array}$ \\
\hline 16 & $\begin{array}{l}\text { Cobertura de inmunización contra el neumococo en niños y niñas } \\
\text { de } 1 \text { año }\end{array}$ \\
\hline 17 & $\begin{array}{l}\text { Cobertura de inmunización contra la Triple viral en niños y niñas } \\
\text { de un año }\end{array}$ \\
\hline 18 & $\begin{array}{l}\text { Cobertura de inmunización contra la influenza en niños y niñas } \\
\text { menores de } 1 \text { año }\end{array}$ \\
\hline
\end{tabular}


19 Porcentaje de mujeres gestantes que asistieron a control prenatal y que se practicaron la prueba de VIH (Elisa)

20 Tasa de transmisión materno infantil de VIH

21 Porcentaje de embarazos en mujeres adolescentes

22 Porcentaje de mujeres gestantes con sífilis que han sido diagnosticadas y tratadas antes de la semana 17

23 Cinco primeras causas de morbilidad en menores de 5 años

24 Tasa de morbilidad por EDA (Enfermedad Diarreica Aguda) en menores de 5 años

25 Tasa de morbilidad por ERA (Enfermedad Respiratoria Aguda) en menores de 5 años

26 Porcentaje de niños, niñas entre 0 y 10 años que asisten a controles de crecimiento y desarrollo

27 Tasa de sífilis congénita

Desarrollo del sistema de información aplicando los procesos de desarrollo TSPi y PSP, en un proyecto real con la participación de 17 estudiantes de octavo semestre de la Universidad de Caldas del programa ingeniería de sistemas y computación.

Aunque TSPi desarrolla productos en varios ciclos para crear un producto final, el sistema de indicadores SAN se dividió en tres subsistemas y para cada subsistema se realizó sólo un ciclo de desarrollo, los subsistemas se desarrollaron de forma paralela y al final se realizó la integración del sistema.

Los estudiantes registraron los datos en la herramienta Procces Dashboard que soporta los scripts, formularios y plantillas de PSP, a la vez que automatiza la recolección de métricas y su análisis [16].

Se construye la propuesta de mejora del desarrollo del sistema de información, donde se presentan los problemas presentados, el análisis de cada uno de los roles, la participación de cada integrante en el proyecto y mejoras y métodos a tener en cuenta en el desarrollo de software en equipo.

\section{CONCLUSIONES}

Se realizó el estudio de los indicadores de SAN a nivel internacional, nacional y departamental, se presentaron los indicadores al grupo de expertos del OBSAN, quienes realizaron un consenso de los indicadores a los que les haría seguimiento, concluyendo que aunque muchos de estos indicadores son importantes en el ámbito de SAN es necesario cumplir con los 27 indicadores que hacen parte de la política pública, ya que son exigidos y vigilados por la Procuraduría General de la Nación y que para poder incluir otros indicadores es necesario contar con mayor infraestructura y presupuesto.

El desarrollo del sistema realizado con los estudiantes mostró una serie de desafíos asociados con el aprendizaje de los procesos software TSPi y PSP, entendiendo que desarrollar software es un proceso complejo con un conjunto de actividades de ingeniería que requiere técnicas para construir software que van más allá de la codificación de programas. El trabajo realizado en la asignatura se centró en desarrollar habilidades en Ingeniería del Software que involucró planeación del proyecto, la administración y gestión del tiempo, el manejo y la gestión de los defectos, la estimación del tamaño del producto, el trabajo individual, el trabajo en equipo y la propuesta de mejora.
La práctica, generó un impacto positivo, tanto para el gestor del proyecto como para los estudiantes, la experiencia fue positiva y se constituye en un aporte para darle continuidad a procesos experimentales con estudiantes.

Se entregó el sistema de información indicadores SAN de Caldas al OBSAN, buscando con ello contribuir al mejoramiento de la seguridad alimentaria y nutricional de la población Caldense.

\section{AGRADECIMIENTO}

A los Integrantes de la Red SAN del departamento de Caldas, especialmente a la Doctora Olga Chávez, al ingeniero Fernando Castellanos Coordinador del OBSAN y Docente de la Universidad de Caldas, Doctor Carlos Cuesta Director de Tesis, estudiantes de la Universidad de Caldas que participaron en el proyecto, al Doctor Mauricio Alba Director de la Maestría Gestión y Desarrollo de Proyectos de Software de la Universidad Autónoma de Manizales.

\section{REFERENCIAS}

[1] Organización de las Naciones Unidas Para la Alimentación y la Agricultura. FAO, Plan de Acción Sobre la Cumbre Mundial de la Alimentación. $\quad$ Roma. $1996 . \quad$ Disponible en:http://www.fao.org/docrep/003/w3613s/w3613s00.htm

[2] W. Humphrey, "Introduction to Team Software Process", SEI Series in Software Engineering: Addison Wesley, 2000.

[3] W. Humphrey, "The Personal Software Process". CMU/SEI: Addison Wesley, 2000.

[4] J. Landeta, "Una Técnica de Previsión para la Incertidumbre". El método Delphi: Ariel. Barcelona, 1999.

[5] Organización de las Naciones Unidas Para la Alimentación y la Agricultura - FAO, "Indicadores Básicos Propuestos para Vigilar de la Seguridad Alimentaria". Disponible en: http://www.fao.org/docrep/meeting/x8228s.htm.

[6] Procuraduría General de la Nación, "Indicadores Sociales". Disponible en: https://www.procuraduria.gov.co/portal/ infancia_indicadores.page.

[7] C. Laman, "UML y Patrones Introducción al Análisis y Diseño Orientado a Objetos y al proceso unificado". Segunda Edición, México: Pearson Educación, 2003.

[8] W. Humphrey," Introduction To The Team Software Process: Addison Wesley", 2010.

[9] Procuraduría General de la Nación, "Indicadores Sociales". Disponible en: http://www.procuraduria.gov.co/ portal/infancia_indicadores.page.

[10] Gobernación de Caldas, Rendición Pública de Cuentas, Garantía de Derechos: Infancia, Niñez y Adolescencia; Tomo I. Manizales, 2011.

[11] Gobernación de Caldas, Rendición Pública de Cuentas, Garantía de Derechos: Infancia, Niñez y Adolescencia: Tomo II. Manizales, 2011.

[12] Gobernación de Caldas, Rendición Pública de Cuentas, Garantía de Derechos: Infancia, Niñez y Adolescencia: Tomo III. Manizales, 2011.

[13] Concejo Nacional de Política Economía Social, Política Nacional de Seguridad Alimentaria y Nutricional: Departamento Nacional de Planeación. Bogotá, 2008.

[14] Gobernación de Caldas, Construyendo Seguridad Alimentaria y Nutricional en Caldas Desde lo Municipal, Una Apuesta para la Superación del Hambre y la Pobreza: Plan Departamental de 
Seguridad Alimentaria y Nutricional de Caldas 2007-2017, Manizales, 2008.

[15] "Ingeniería de Software", Listas de Chequeo. Facultad de Ingeniería, Universidad Autónoma de Manizales, 2010.

[16] Carnegie Mellon University, "The Software Process Dashboard initiative". Disponible en: http://www.processdash.com.2009

[17] Diario Oficial 46446 de la República de Colombia, "Ley N 1098", Bogotá DC, noviembre de 2006.

[18] G. Booch, I. Jacobson, J. Rumbaugh, El Lenguaje Unificado de Modelado, Manual de Referencia. Segunda Edición. Madrid: Pearson Educación. 2007.

[19] W. Humphrey, "PSP A Self-Improvement Process for Software Engineers". Addison Wesley, (2005).

[20] C. Larman, UML y Patrones Introducción al Análisis y Diseño Orientado a Objetos. México: Pearson Educación. 1999.

[21] G. Booch, I. Jacobson, J. Rumbaugh. The Unified Modeling Language, Reference Manual. Readin, MA: Addison Wesley. 1999.

[22] Organización de las Naciones Unidas para la Alimentación y la Agricultura. FAO, "Indicadores de Nutrición para el Desarrollo. IRD Institut de Recherchepour le Développement Montpellier, Francia. Servicio de Planificación, Estimación y Evaluación de la Nutrición Dirección de Nutrición y Protección del Consumidor. Roma. Disponible en: http://www.fao.org/3/a-y5773s.pdf

[23] Organización de las Naciones Unidas para la Alimentación y la Agricultura. FAO, Conclusiones de la Mesa Redonda Destinada a Revisar los Métodos Empleados para Calcular el Número de Personas Hambrientas. Roma. Disponible en: http://www.fao.org/bodies/cfs/cfs37/es/

[24] Organización de las Naciones Unidas para la Alimentación y la Agricultura -FAO, Sistema Informático en Internet para Estadísticas Nacionales y Subnacionales de Alimentos y Agricultura -CountrySTAT: División Estadística. Roma. Disponible en: http://www.fao.org/economic/ess/ess-capacity/countrystat/es/

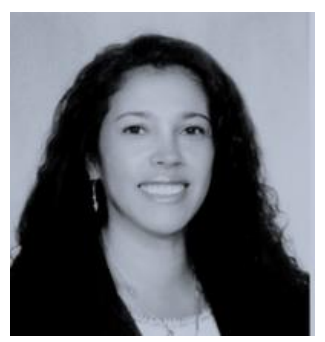

Derly Patricia Soto Alzate, Ingeniera de Sistemas de la Universidad Antonio Nariño de Pereira, Especialista en Ingeniería de Software de la Universidad Autónoma de Manizales, Especialista en Alta Gerencia en Economía Pública de la Escuela Superior de Administración Pública (ESAP), Magister en Gestión y Desarrollo de Proyectos de Software de la Universidad Autónoma de Manizales. Trabajó como docente en la Universidad de Caldas desde el año 2001 hasta el año 2013. Investigadora del proyecto "Impacto del uso de tecnologías informáticas en el análisis y clasificación de la taxonomía de los insectos" de la misma universidad, Docente del programa de Administración de Sistemas de la Fundación Universitaria Comfamiliar desde el año 2018.

ORCID: https://orcid.org/0000-0002-3664-4460 